\title{
Competing Morbidities In Advanced Head And Neck Squamous Cell Carcinoma Concurrent Chemoradiotherapy: A Strong Implication Of A Multidisciplinary Team Approach
}

This article was published in the following Dove Press journal:

Cancer Management and Research

\author{
Grazia Lazzari ${ }^{1}$ \\ Maria Assunta De Cillis ${ }^{2}$ \\ Giovanni Buccoliero ${ }^{3}$ \\ Giovanni Silvano ID \\ 'Radiation Oncology Unit, S. Giuseppe \\ Moscati Hospital, Taranto 74I00, Italy; \\ ${ }^{2}$ Otorynolaringoiatry Unit, S. Giuseppe \\ Moscati Hospital, Taranto 74I00, Italy; \\ ${ }^{3}$ Infection Diseases Unit, S. Giuseppe \\ Moscati Hospital, Taranto 74I00, Italy
}

\begin{abstract}
Concurrent chemoradiotherapy (CCRT) is the standard approach for the treatment of locally advanced head and neck squamous cell carcinoma. Despite its undisputed advantages, CCRT is associated with acute and late toxicities, leading to unfavorable implications (eg, unplanned interruptions and noncancer-related mortality). The former prolongs the overall treatment time leading to a detrimental effect on tumor control. The latter consists of several noncancer morbidities arising from treatmentrelated toxicities, identifying a new pathway in cancer fate. This pathway has been termed noncancer mortality or competing mortality and consists of a series of treatment-competing morbidities, which nullify all therapeutic efforts aimed at curing these patients. The management of patients with head and neck squamous cell carcinoma who experience treatment-related toxicities is complex and requires expertise in oncological treatment as well as supportive care. The optimal management of these patients should start with knowledge regarding the most important competing morbidities developing during all phases of the disease (ie, from diagnosis to follow-up) to minimize treatment interruptions, ensure appropriate psychological support, and achieve the best oncological result. The purpose of the present review is to analyze the most important competing morbidities due to patient's condition at baseline and CCRT, which could result in noncancer mortality. A multidisciplinary team approach is strongly required in the management of this disease.
\end{abstract}

Keywords: competing mortality, head and neck squamous cell carcinoma, concurrent chemoradiotherapy, multidisciplinary team

\section{Introduction}

Based on meta-analysis and randomized studies, concurrent chemoradiotherapy (CCRT) is currently the standard care for locally advanced head and neck squamous cell carcinoma (HNSCC), ${ }^{1}$ resulting in the highest laryngectomy-free survival rate, best locoregional control rate, and low distant metastasis rate versus radiotherapy alone. ${ }^{2}$ In terms of drugs, platinum-based CCRT has emerged as the principal treatment of choice, ${ }^{3}$ despite the availability of other novel and effective agents. ${ }^{4}$ However, CCRT is associated with the development of acute and late adverse effects, which may lead to a competing morbidity pathway that reduces overall survival (OS) and decreases long-term disease-free survival (DFS).
Correspondence: Grazia Lazzari

Radiation Oncology Unit, S. Giuseppe

Moscati Hospital, Str. Per Martina Franca,

Taranto 74100, Italy

Tel +039-099 458572I

Email lazzarigrazia@gmail.com 
Therefore, strict selection of patients eligible for this type of therapy is crucially important. It has been estimated that this competing morbidity pathway is present in $\leq 50 \%$ of the treated patients. ${ }^{5}$ The first report regarding these unintended effects was recently published by Forastiere et al, analyzing the long-term results of the Intergroup Radiation Therapy Oncology Group 91-11 study. This exploratory analysis showed that the rate of deaths not attributed to larynx cancer or treatment was higher in patients receiving concomitant chemotherapy (ie, induction chemotherapy and radiotherapy alone $30.8 \%$ vs $20.8 \%$ vs $16.9 \%$, respectively). ${ }^{6}$ This offers a basis for thought from a radiobiological and clinical point of view. It is well acknowledged that toxicities exert a detrimental effect on local control. Treatment toxicity is one of the major causes of unplanned interruptions, which leads to a prolongation of the overall treatment time. The radiobiological consequence is the reinitiation of an accelerated repopulation of tumor clones, which reduces local control and increases toxicity. ${ }^{7}$ Following an unplanned interruption, an increased radiation dose administered over a short period of time is required to compensate for the gap in treatment. The increased dose required to maintain a constant control rate in HNSCC has been calculated at $0.5-0.6 \mathrm{~Gy} /$ day using 2 Gy/fraction. However, this treatment compensation may result in toxicity. ${ }^{8}$ In addition, local irradiation and systemic effects are strongly interlinked. Russi et al have described in detail the impact of local irradiation on the whole organism through several inflammatory steps. ${ }^{9}$ The authors clearly explained that local irradiation leads to mucosal injury that perturbs the organism as a whole through cytokine cascades, generating an abscopal inflammatory effect on distant organs. As a result, several occasionally life-threatening systemic syndromes or organ failures may occur following CCRT. Knowledge of the systemic consequences related to CCRT together with the conditions of the patients with HNSCC at baseline and during treatment are of crucial importance, highlighting the need for a multidisciplinary team (MDT) approach.

\section{Patient-Related Factors}

Evaluation of the physical conditions of patients with HNSCC at baseline is the first process for the identification of factors which could impact the occurrence of toxicities and final outcome. This process influences the selection of treatment and therapeutic process prior to and during treatment. Thus far, the age of patients with HNSCC and their nutritional status have been identified as important factors.

\section{Advanced Age And Comorbidities}

Elderly patients represent approximately half of the HNSCC diagnoses. This is a particularly challenging population associated with reduced treatment compliance. Thus, the evaluation of frailty due to advanced age and any other associated comorbidities is crucial in the decision to prescribe CCRT that could benefit the patients in terms of survival. A meta-analysis revealed that patients aged $>70$ years have no survival benefit following the addition of chemotherapy to radiation. ${ }^{1} \mathrm{Kwon}$ et al recently conducted an analysis with regard to patients enrolled in specific clinical trials. The results confirmed that elderly patients with HNSCC are less fit for CCRT versus younger patients due to the high incidence of additional comorbidities, which lead to correspondingly higher rates of noncancerrelated mortality. ${ }^{10} \mathrm{~A}$ single-institution retrospective study involving 369 elderly patients analyzed the compliance to CCRT, underlining some differences in outcome between patients aged $\geq 70$ years or $<70$ years. In summary, it was reported that patients aged $\geq 70$ years were treated less often with thrice-weekly cisplatin $(25.5 \%$ vs $71.4 \%$, respectively) and more often with weekly carboplatin (31.9\% vs $3.4 \%$, respectively) than those aged $<70$ years $(p<0.001)$. Notably, patients aged $\geq 70$ years exhibited a higher rate of hospitalizations due to toxicities $(36.2 \%$ vs $21.1 \%$, respectively; $\mathrm{p}=0.02$ ). Furthermore, the same group of patients had an increased risk of death at 3 months following CCRT $(p=0.005)$ and worse survival over time $(p=0.002) .{ }^{11}$ Comorbidity has been found to be an independent predictor of short-term mortality and OS in patients with HNSCC. ${ }^{12}$ However, treating physicians may not integrate this feature in their decisional process, ${ }^{13}$ despite the fact that at least mild comorbidity has been documented in $\leq 54 \%$ of the patients with HNSCC at the time of diagnosis. ${ }^{14}$ In clinical practice, the performance status is routinely assessed during enrolment in the CCRT protocol; however, this approach seems inadequate for evaluating comorbidities and predicting the risk of a noncancer mortality. The most widely used and validated model in oncology for the evaluation of comorbidities is the Charlson Comorbidity Index, assessing the risk of treatment-related toxicity and its outcome through intensification of therapy. ${ }^{15}$ Furthermore, other systems for the scoring of comorbidities, such as the Adult Comorbidity 
Evaluation-27, Cumulative Illness Rating Scale for Geriatrics, Geriatric (G8), Cancer and Aging Research Group, and Generalized Competing Event scores, have been used in elderly patients with HNSCC. More recently, all these models have been incorporated into an online tool to calculate indices of comorbidity in patients with head and neck cancer (HNSCC), showing a high degree of reproducibility and reliability. ${ }^{16}$

\section{Glasgow Prognostic Score (GPS)}

This score summarizes the inflammatory and metabolic activity of cancer hidden in the patient. It takes into account two parameters, namely the plasma levels of C-reactive protein (CRP) and the serum levels of albumin. The former is related to the inflammatory state induced by the tumor, while the latter is an expression of the nutritional state of the patient modified by cancer catabolism. Several reports have demonstrated that the presence of a systemic inflammatory response is associated with increased weight loss (WL), loss of lean tissue, and a functional decline, leading to cancer-related cachexia. ${ }^{17}$ This type of cachexia is associated with complex metabolic, as well as molecular and cellular alterations defining an inflammatory status also represented by elevated plasma levels of CRP. ${ }^{18}$ Moreover, the levels of serum albumin are correlated with the systemic inflammatory condition of the patient through several pro-inflammatory cytokine cascades. ${ }^{19}$ Thus, increased catabolism, chronic malnutrition, and inflammatory reactions due to cancer lead to hypoalbuminemia, which has been associated with poor survival in various types of cancer. The combination of serum CRP and albumin concentrations defines the modified GPS (mGPS) that has been adopted as a prognostic therapeutic tool in a wide variety of cancers. ${ }^{20}$ Therefore, it should be considered an objective prognostic nutritional marker in HNSCC treated with CCRT as a survival prognosticator at baseline and during all the phases of CCRT. In fact, the mGPS may change over time. Changes in the mGPS score prior to, during, and after CCRT have been identified as important prognosticators of DFS and OS. Patients exhibiting an improved GPS at the end of treatment have been linked to better OS $(p=0.013)$ and relapse-free survival $(p=0.012)$ compared with those yielding a worsened final GPS. ${ }^{21}$

\section{Weight Loss}

In all cancer patients, weight loss (WL) is considered an involuntary effect mainly attributed to cancer-induced catabolism, which impacts loss of fat-free mass. ${ }^{22}$ In particular, in HNSCC patients, WL seems to be increased by additional factors. One of these factors is the tumor primary site in oro-hypopharyngeal cancers, which are characterized by a predominant mechanical impairment of the ability to eat. ${ }^{23}$ Swallowing problems caused by the obstruction of the passage of bolus by the tumor, metabolic alterations affecting appetite, and radiation-related mucositis on the treated area are also factors to consider. ${ }^{24}$ The impact of these factors on WL has been widely assessed in the past. In particular, in the ARTSCAN study, the oropharyngeal tumor site, age $>65$ years, underweight, absence of tube feeding, Karnofsky performance status $<80 \%$, and swallowing problems reported at the initiation of treatment have been significantly related to WL. ${ }^{25}$ Moreover, the trend of WL during and after the treatment completion strongly affects the final outcome. It has been recognized that a reduction of the pre-diagnosis weight by $>20 \%$ is significantly related to poor survival during CCRT and within 30 days after treatment completion. ${ }^{26}$ Furthermore, it should be considered that WL during CCRT is not a static but a variable feature that changes during treatment. During CCRT, $55 \%$ of the patients may lose an additional $10 \%$ or more of their body weight. In the acute phase, this loss has been measured to be $11.3 \%$ and continues to decrease after RT completion, with a nadir recorded at 5 months after the termination of RT. ${ }^{25}$

This trend over time may be a useful finding for adopting prompt and customized nutritional interventions prior to, during, and after treatment. Routine nutritional screening is considered an essential component of modern cancer care; however, the early assessment of the risk of malnutrition has not been well established. The Malnutrition Universal Screening Tool has shown its strength for application to adult patients across all healthcare settings, including oncology. Taking into account the WL linked to radiation oncology, the Malnutrition Universal Screening Tool appears to be effective in detecting the risk of WL in this subset of patients. Moreover, it is a simple and rapid method that can be applied by any healthcare professional, with a high validity for early screening. Ideally, this method should antedate a comprehensive nutritional assessment and guide intervention. ${ }^{27}$

\section{Nutritional Intervention Issues}

WL is considered the main surrogate of nutritional status impairment in CCRT. Severe malnutrition, defined as WL $>10 \%$ within 6 months preceding primary tumor diagnosis 
has been found as an independent factor affecting the OS of primary HNSCC patients. During treatment, WL exerts a negative effect on OS, leading to unplanned interruptions in treatment or prolonged hospitalization. Discontinuation of RT for $>5$ days has been reported in $53 \%$ of the patients with a WL $>20 \%$ during CCRT, with a complete interruption observed in $29 \%$ of those patients. ${ }^{28}$ After completion of treatment, WL (an additional 10\%) can continue further due to the persistence of severe mucositis and dysphagia. Thus, the implementation of a customized nutritional program is required. Several international guidelines suggest that intensive nutritional counseling and oral nutritional supplements should be applied to increase dietary intake, as well as prevent therapy-associated WL and interruption of RT in patients with HNSCC undergoing CCRT. ${ }^{29-31}$ Paccagnella et al documented that patients with HNSCC who were enrolled in the nutritional intervention policy had lost significantly less weight than those in the control group (CG) who did not participate in the nutritional program $(\mathrm{p}=0.024)$. As a result, patients enrolled in the nutritional intervention policy exhibited WL at the end of treatment $(-4.6 \%)$, followed by prompt weight regain. On the contrary, CG patients continued to lose weight for 6 months after the end of CCRT. In this group, statistical significance was found for the proportion of patients who had RT interruptions for $>5$ days due to toxicity $(\mathrm{p}=0.007)$, delay of RT (days) due to toxicity ( $\mathrm{p}=0.038$ ), and the proportion of patients admitted to a hospital for mucositis $(\mathrm{p}=0.030) .{ }^{32}$ As shown by Hughes et al, the implementation of the WL risk stratification along with appropriate guidelines concerning the nutritional status of patients with head and neck cancer has been demonstrated to be a health and cost-effective strategy. ${ }^{33}$

\section{Caloric Intake Interventions}

In patients receiving RT or CCRT, the total energy expenditure and protein requirement have been estimated to be $30-35 \mathrm{kcal} / \mathrm{kg} /$ day and $1.2 \mathrm{~g} / \mathrm{kg} /$ day, respectively. ${ }^{34}$ For the minimization of WL, the nutritional support should be promptly performed in the following scenarios: a body mass index (BMI) $<18.5 \mathrm{~kg} / \mathrm{m}^{2}$, an unintentional $\mathrm{WL}$ $>10 \%$ over 3-6 months, a BMI $<20 \mathrm{~kg} / \mathrm{m}^{2}$, a minimal intake $<5$ days, or increased nutritional requirements due to catabolism. Three main methods of nutritional support have been identified, namely oral, parenteral, and enteral. Of note, enteral nutrition is preferred for patients with HNSCC receiving CCRT. ${ }^{35}$ The type and volume of enteral nutrition depend on the patient's symptoms and current intake because they are likely to change throughout and following treatment. Hence, a range of nutritionally polymeric complete feeds have become available to customize and ensure an appropriate caloric intake. ${ }^{36}$

\section{Enteral Nutrition: Percutaneous Gastrostomy (PEG) Or Nasogastric Tube (NGT) Feedings?}

Enteral nutrition is the preferred approach to improving the nutritional status of patients with HNSCC receiving CCRT $^{37}$ The strategies employed for nutritional supply during enteral feeding are the NGT and PEG. At present, there is insufficient evidence to determine which of these two strategies is the optimal method for these patients, as both have demonstrated effectiveness in improving nutritional intake. ${ }^{38}$ Several studies have shown that at 6 weeks following the end of radical chemoradiation, the patients with PEG feeding had lost significantly less weight than those with NGT feeding; however, 6 months later, the difference in weight was not significant. ${ }^{39}$ Furthermore, a retrospective study indicated that enteral feeding did not influence WL at the end of treatment or unscheduled interruptions of RT. ${ }^{40}$ Nevertheless, a systematic review of PEG and NGT feedings revealed equivalent outcomes in terms of maintaining weight, rate of infections, difference in survival, duration of feeding, and days of delay in $\mathrm{RT}^{41}$ Another factor which could influence the choice of feeding approach is the duration of use of these devices, considering that prolonged use of NGT feeding is associated with decubitus ulcerations of the mucosa of the nose and pharynx. Concerning this issue, the National English Institute for Health and Care Excellence guidelines on enteral feeding suggest that gastrostomy should be performed in patients requiring enteral feeding for $>4$ weeks. $^{36}$

\section{Prophylactic PEG (P-FT) Or Reactive PEG (R-FT)?}

A controversial topic is the use of P-FT or R-FT, whereas this last occurs when patients are unable to meet their nutritional requirements through the intake of oral nutritional supplements during treatment. Several retrospective and few prospective studies have addressed this topic by comparing these two approaches. However, as investigated by Bossola in a narrative review, there is no definitive conclusion drawn owing to the similar nutritional outcomes observed with both approaches. ${ }^{42}$ Particularly, in a study conducted by Lewis et al, the number of 
interruptions of treatment was similar although patients with P-FT had completed a greater number of chemotherapy cycles compared with those receiving R-FT $(\mathrm{p}<0.001)$. Notably, there were no differences found in terms of OS and DFS. ${ }^{43}$ However, in another study, patients who received R-FT had a significantly higher stricture rate and aspiration rate compared with those in the P-FT group. In addition, significantly fewer hospitalizations were observed in the P-FT group versus the R-FT group. Overall, when accounting for both PEG placement and hospitalizations, the prophylactic approach was found to be more cost-effective. ${ }^{44}$ An Australian group has proposed the implementation of a nutritional program based on WL risk stratification guidelines. ${ }^{33}$ This program consists of an algorithm, which includes the early identification and insertion of a P-FT tube in HNSCC patients with high-risk WL. This active implementation of customized nutritional guidelines according to the WL risk has resulted in a significant decrease in the number of hospital admissions and duration of hospital stay. ${ }^{33}$ Strom et al indicated some factors, such as a BMI $<25$, accelerated irradiation fractionation, a tumor $\mathrm{T}$ classification of $\geq 3$, and a cumulative cisplatin dose $>200 \mathrm{mg} / \mathrm{m}^{2}$, that can be used to determine the appropriate time for the reactive placement of a PEG tube during CCRT in patients with symptomatic oropharyngeal cancer. ${ }^{45}$ However, there is insufficient evidence to determine the optimal method of enteral feeding. The use of routine swallowing tests performed at baseline and during treatment could be a useful method for the early identification of symptomatic patients.

\section{Refeeding Syndrome And Eating Rehabilitation}

Refeeding syndrome is a metabolic disturbance occurring with the reintroduction of nutrition in patients who are severely malnourished irrespective of the feeding route. The main feature of this syndrome is hypophosphatemia, with an abnormal sodium and fluid balance. Changes in glucose, protein, fat metabolism, as well as thiamine deficiency, hypokalemia, and hypomagnesemia are also accounted. ${ }^{36}$ Prior to the initiation of feeding, intake of thiamine and multivitamin complex is required for patients at risk, followed by a slow-feeding supplement, and careful rehydration. Importantly, attention is paid to the correct levels of potassium, phosphate, and magnesium. Implementation of a rehabilitation eating program is mandatory after discontinuation of feeding. Dietitians can play a key role in this process by offering tailored healthy eating advice that takes into consideration the long-term side effects experienced by the treated patients. As emphasized by Talwar et al, ${ }^{35}$ a nutritional intervention (dietary counseling and/or supplements) should be offered for $\leq 3$ months after treatment. The rehabilitation program recommends that patients should be observed fortnightly for $\geq 6$ weeks post treatment and reviewed by the dietitian for $\leq 6$ months or as long as they require management of chronic toxicities, WL, or tube feeding.

\section{Fatigue}

Fatigue has been described as the most distressing symptom experienced by patients with cancer during treatment, especially with the addition of RT. ${ }^{46}$ Fatigue exerts a negative effect on the compliance and management of therapy, influencing the decision to interrupt the treatment or decrease the dose. As shown by Hickok et al, although it is not a specific symptom, the degree of fatigue appears to vary according to the irradiated anatomical site. ${ }^{47}$ In that study, compared with other cancer sites, patients with HNSCC showed a higher frequency and severity of fatigue at baseline and throughout the 5-week treatment period. Moreover, persistence of fatigue throughout the entire course of RT has been observed in nearly $52 \%$ of the patients. Several questionnaires have been developed for assessing the level of fatigue. Among them, the Multidimensional Fatigue Inventory-20 has been validated as a useful tool in oncological research. ${ }^{48}$ Jereczek-Fossa et al showed that the level of fatigue increased during RT, with the maximum noted at week 6 and gradually decreasing thereafter. The multivariate analysis revealed that advanced age, psychologic disorders, and previous head and neck surgery correlated with a higher pre-RT level of fatigue. In turn, the pre-RT fatigue score $(\mathrm{p}<0.0001)$, induction and/or CCRT ( $\mathrm{p}=0.035)$, and need of cortisone during RT $(\mathrm{p}<0.005)$ also correlated with a higher level of fatigue during RT. Furthermore, the pre-RT fatigue score $(\mathrm{p}<0.0001)$, induction and/or concomitant computed tomography $(\mathrm{p}<0.001)$, and the need of cortisone during RT ( $>0.005)$ were also related to a higher level of fatigue post-RT. ${ }^{48}$ Although they may be concurrently present in the patient, fatigue is not a surrogate feature of WL or anemia. A low-level hemoglobin (Hgb) pre-RT has been associated with a high level of RT-induced fatigue in some studies. ${ }^{49}$ However, in other studies, fatigue was primarily associated with the Karnofsky performance status and psychological distress. ${ }^{50}$ The correction of anemia prior 
to or during RT could be helpful when fatigue is accompanied by a low level of $\mathrm{Hgb}^{51}$ Currently, the role of erythropoietin in the correction anemia and fatigue in these patients remains unclear, as documented by a prospective randomized trial using epoetin alfa. ${ }^{52}$ This study showed that administration of erythropoietin in patients with HNSCC receiving CCRT ameliorated the level of $\mathrm{Hgb}$, whereas it did not affect fatigue on any subscale or overall score.

\section{Cancer-Related Anemia}

Anemia is a condition caused by a decrease in the concentration of red cells or level of Hgb in peripheral blood leading to a reduction in the oxygen-carrying capacity of the blood and hypoxia in tissues. ${ }^{53}$ The pathophysiology of anemia in cancer is multifactorial, including factors related to the cancer per se and treatment side effects. Among the tumor-associated factors, the combination of tumor bleeding and low nutritional oral intake with deficiency in folic acid and vitamin B12 has been linked to an inflammatory anticancer immune response. ${ }^{54}$ Considering this, the interaction between the tumor cell population and immune system leads to the release of cytokines, such as interferon gamma, interleukin-1, and tumor necrosis factor- $\alpha$. The consequence is an inflammatory state, which suppresses the differentiation of erythroid precursor cells in the bone marrow enhanced by the activation of macrophages. In turn, this effect leads to a shorter erythrocyte half-life and a decrease in iron utilization, achieving high levels of erythropoietin and ferritin with sideropenic anemia. ${ }^{55} \mathrm{~A}$ low level of Hgb has been shown to be detrimental. ${ }^{56}$ Several previous studies have reported thresholds for the low level of $\mathrm{Hgb}$ ranging from $9 \mathrm{~g} / \mathrm{dL}$ to $14.5 \mathrm{~g} / \mathrm{dL} .{ }^{56}$ Hence, thus far, this threshold remains unclear. An association between lower baseline Hgb and worst treatment outcome was confirmed by a secondary analysis of two European randomized trials (ARO 95-06, SAKK 10/94). A lower baseline $\mathrm{Hgb}$ was associated with decreased OS in patients who received CCRT $(\mathrm{p}=0.009)$ and in those who underwent RT alone $(\mathrm{p}<0.001) .{ }^{57}$ Furthermore, studies investigating the quality of life of patients suggested that correction of moderately severe anemia may result in significant gains. However, the use of erythropoiesis-stimulating agents (ESAs) in patients with HNSCC remains controversial on the basis of the unfavorable outcomes, as stated by several studies (eg, ENHANCE, DAHANCA 10, RTOG 99-03, and an erythropoietin alfa study). ${ }^{52,58-60}$ All these studies confirmed that correction of the level of Hgb using ESAs during RT in patients with HNSCC has resulted in a significantly poorer tumor control and survival.

\section{Correction Of Anemia}

According to the available guidelines, the amelioration of symptoms and avoidance of transfusion therapy are the main indications for the administration of erythropoietin, although transfusion of red blood cells is also an option. The European Organization for Research and Treatment of Cancer updated their guidelines regarding the use of ESAs stating that they reduce the number of transfusions and significantly improve quality of life in patients with chemotherapy-induced anemia. A sustained Hgb level of approximately $12 \mathrm{~g} / \mathrm{dL}$ has been recommended as the target of treatment with ESAs. ${ }^{61}$ Furthermore, based on the detrimental outcomes reported in several trials, the guidelines established by the American Society of Clinical OncologyAmerican Society of Hematology recommend the administration of erythropoietin in patients with chemotherapyassociated anemia whose cancer treatment is not curative in intent and their $\mathrm{Hgb}$ has declined to $10 \mathrm{~g} / \mathrm{dL}^{62}$

\section{Treatment-Related Systemic Morbidities}

Approximately $50 \%$ of the patients with $\mathrm{HNC}$ receiving CCRT are at risk of developing treatment-related toxicity, which negatively impacts the outcome. Herein, the most important and frequently recorded toxicities reported in clinical practice are discussed.

\section{Cisplatin-Induced Toxicity}

In HNSCC, cisplatin is currently the most widely used cytotoxic agent in combination with radiation. A meta-analysis of chemotherapy in HNC showed a $65 \%$ benefit at 5 years in patients receiving concomitant chemotherapy. Moreover, among monochemotherapies in clinical trials, platinumbased regimens were found to be more effective than other drugs. ${ }^{1}$ The rationale for adding cisplatin to $\mathrm{RT}$ is based on its radiosensitizing properties coupled with a toxicity profile not overlapping with RT. Administration schedules of $100 \mathrm{mg} / \mathrm{m}^{2}$ thrice weekly or weekly $40 \mathrm{mg} / \mathrm{m}^{2}$ are usually used, ${ }^{63,64}$ while a new alternative weekly administration of $20 \mathrm{mg} / \mathrm{m}^{2}$ for 5 days every 4 weeks has been recommended owing to a favorable safety profile. ${ }^{65}$ Apart from the schedule, the cumulative dose of cisplatin in concurrent chemoradiation protocols for HNSCC seems to be significantly related with survival $(\mathrm{p}=0.027)$, suggesting that higher doses of cisplatin are associated with greater benefit. ${ }^{66}$ 
Thus, the recommended cumulative dose of cisplatin administered during RT should be $\geq 200 \mathrm{mg} / \mathrm{m}^{2}$. However, this approach is linked to toxicity (eg, myelosuppression, neurotoxicity, ototoxicity, and nephrotoxicity) and reduced compliance to the planned regimen of chemotherapy (ie, 49-61\%), as shown in several trials. ${ }^{2,67,68}$ Cisplatin belongs to the group of agents with a potentially high emetogenic risk, in which the frequency of emesis is estimated to be $>90 \%{ }^{69}$ Therefore, according to the available antiemesis guidelines established by the National Comprehensive Cancer Network V2.2018.2018, ${ }^{70}$ the Multinational Association for Supportive Care in Cancer, the European Society of Medical Oncology, and the American Society of Clinical Oncology, adequate antiemetic prophylaxis is required in patients receiving treatment with cisplatin. ${ }^{71,72}$

\section{Cisplatin-Induced Nephrotoxicity}

Acute platinum-induced nephrotoxicity occurs in one-third of patients nearly 10 days after its supply, as an expression of damage to kidney tubule cells. In fact, it is associated with the inability to concentrate urine rather than a reduction in urinary output, resulting in hypovolemia and irreversible kidney failure. The mechanism consists of tubular damage occurring mainly in the S3 part of the tubular proximal loop, Henle loop, and distal tubular district via tumor necrosis factor- $\alpha$ effect cytokine cascade associated with an immune inflammation. The results are glucosuria, aminoaciduria, magnesium loss, and anemia due to renal damage and impairment in the production of erythropoietin. ${ }^{73}$ The scales used to measure nephrotoxicity impact on its real incidence, explaining the observed controversial toxicity data. According to the Common Terminology Criteria for Adverse Events criteria grade 3-4, the incidence of acute nephrotoxicity varies between $1 \%$ and $46 \% .^{2-65}$ Nevertheless, the incidence of nephrotoxicity could be increased to nearly $53 \%$ when measured using the RIFLE scale (Risk, Injury, Failure, Loss of kidney function, End-stage kidney disease), as found by Espeli et al. ${ }^{64}$ However, in clinical practice, the measurement of nephrotoxicity is usually based on the rate of glomerular filtration, using the Common Terminology Criteria for Adverse Events v4.0. Through this method, grade 2 nephrotoxicity based on a glomerular filtration rate of $30-59 \mathrm{~mL} / \mathrm{min}$ is considered the main parameter for discontinuing the administration of cisplatin during CCRT. However, grade 2 nephrotoxicity is underreported in the literature. A more useful approach to evaluating renal function is the calculation of the clearance of creatinine using the Cockcroft-Gault formula. Using this method, a retrospective study assessing treatment with thrice-weekly cisplatin CCRT reported grade 2 nephrotoxicity in $25 \%$ of the patients. ${ }^{74}$ This method calculates the rate of glomerular filtration accounting for the age, sex, and body weight. It appears to be a more accurate indicator of renal function over time in patients with HNSCC because their weight and liquid intake are changeable features during CCRT. Different options for minimizing platinum-induced nephrotoxicity have been discussed in the literature. The first option is adjustment of cisplatin schedules, as previously discussed. ${ }^{64,65}$ The role of supportive care based on hydration and forced diuresis with supply of mannitol, furosemide, potassium, and magnesium is fundamental, as indicated by nephron protection protocols. During treatment, loss of body weight or volume depletion predisposes patients to pre-renal hyper-azotemia, which may contribute to acute kidney injury.

\section{Cisplatin-Induced Anemia}

Anemia is the most common hematological side effect in patients with cancer receiving chemotherapy, ${ }^{75}$ exerting a detrimental effect on quality of life and a negative impact on prognosis, with an estimated overall mortality risk of $75 \%$ in HNSCC. ${ }^{76}$ Undoubtedly, anemia in platinum-based chemotherapy is induced by platinum nephrotoxicity through a decrease in the production of endogenous erythropoietin following damage to renal proximal tubular cells. The kidneys are the major source of erythropoietin in adults. Evidence indicated the production of erythropoietin by peritubular interstitial cells, although some studies suggested a renal tubular cell origin. The kidneys accumulate cisplatin to a greater degree than other organs. Moreover, they are the major route for its excretion, leading to a five-fold higher concentration of cisplatin in proximal tubular epithelial cells than in the serum. Thus, the disproportionate accumulation of cisplatin in kidney tissue contributes to cisplatin-induced nephrotoxicity. ${ }^{18}$ Mechanisms identified as major causes of underlying cisplatin-induced renal cell injury include the paradoxical role of gamma-glutamyl-transpeptidase in cisplatin nephrotoxicity, the unexpected ability of proximal tubular cells to metabolize cisplatin to a nephrotoxin, apoptotic pathways, and the presence of reactive oxygen metabolites. ${ }^{18}$ For this reason, cisplatin-associated anemia has been defined as "an erythropoietin deficiency syndrome" promptly responding to the administration of erythropoietin, which corrects the anemia associated with renal failure in the majority of patients. ${ }^{77}$ 


\section{Infections}

Owing to extensive immunosuppression, cancer and its treatment may predispose patients to infections. The array of potential pathogens is wide. However, opportunistic agents and viruses such as the Herpes simplex virus (HSV) or Hepatitis B virus (HBV) should be principally taken into account in addition to conventional microorganisms. Under specific favorable conditions (eg, immunosuppression), infection with any mold, yeast, saprophytic microorganism, or latent virus, may result in a disseminated life-threatening disease. Indwelling catheter, PEG or tube feedings, corticosteroids, and neutropenia-lymphopenia are considered the principal causes. Caution should be taken regarding viruses, such as HSV (Herpes Simplex Virus) and HBV (Hepatitis B Virus). As suggested by several experimental models, local radiation therapy including the temporal lobes in the radiation fields (as in nasopharyngeal cancers) could be responsible for reactivation of latent HSV in the trigeminal ganglion, leading to an increased risk of herpes simplex encephalitis. ${ }^{78}$ Furthermore, patients with chronic HBV infections receiving immunosuppression therapy (eg, oral corticosteroids or chemotherapy), are at risk of $\mathrm{HBV}$ reactivation, developing severe hepatitis, and even life-threatening hepatic failure due to suppression of the immune system and enhancement of virus replication. Prophylactic antiviral therapy using lamivudine should be administered to all HBV-seropositive patients. ${ }^{79}$

\section{Opportunistic Infections}

Among the pathogens associated with opportunistic bacteremia, severe infections by Salmonella are frequent with or without enteritis in immunocompromised patients due to age, malnutrition, use of corticosteroids, or other immunosuppressive therapies. Following oral mucositis with ulcerations and neutropenia, bacteremia may be caused by pathogens normally present in the oral cavity (eg, Rothia dentocariosa, Eikenella corrodens, Stomatococcus mucilaginosus). Bacillus species and Mycobacterium fortuitum are also considered opportunistic agents of catheterrelated bacteremia. Pulmonary infections by Aspergillus and Fusarium have also been described, as well as pneumocystis and Cytomegalovirus pneumonia. ${ }^{80}$

\section{Fungal Infections}

Fungal colonization is the most frequently recorded infection in patients with HNSCC receiving CCRT. The principal source of fungal infections is the mouth flora, skin, and gastrointestinal tract. Predisposing factors are neutropenia, mucositis, and total parenteral nutrition indwelling catheter. However, high-grade (grade III-IV) radiation mucositis has been identified as the most important factor related to fungal colonization and infection in irradiated patients with HNSCC. $^{81}$ The fungal spectrum is wide, including Candida albicans and non-albicans infections. Oral candidiasis, after mucosal colonization by the Candida species, is the most common finding, with albicans as the most frequently isolated organism. Infections with Candida tropicalis and Candida krusei have also been described. ${ }^{82}$ Moreover, infections with Candida parapsilosis have been associated with total parenteral nutrition indwelling catheter delivering total parenteral nutrition. ${ }^{83}$ Furthermore, nonalbicans species (eg, Candida glabrata) have also been implicated in oropharyngeal infections. ${ }^{84}$ Radiation alters the oral environment that predisposes to fungal colonization of the oral mucosa. Therefore, the incidence and severity of mucositis and thrush may be reduced by the prophylactic administration of fluconazole or itraconazole during CCRT. This condition leads to opportunistic candidiasis and occasionally induces fungemia and deep candidiasis in other organs. These effects may be life-threatening in patients with an immunocompromised state. Hence, early diagnosis and management become important in view of the morbidity and lethality associated with oral candidiasis.

\section{Aspiration Pneumonia (AP)}

AP is an underreported complication of CCRT in patients with HNSCC. It has been defined as a pneumonia secondary to inhalation of food particles, saliva, or other foreign substances. Moreover, it represents the major source of post-treatment morbidity, as well as a potential cause of death among HNC patients, with a $42 \%$ increased risk of death. ${ }^{85}$ Significant risk factors related to AP have been identified, such as the absence of tube feeding, advanced clinical stage, incomplete response to treatment, grade 3 dysphagia, and advanced age. ${ }^{86}$ The Surveillance, Epidemiology and End Results-Medicare Database has reported that nearly one-quarter of elderly patients with HNSCC develop AP within 5 years after CCRT. In this population, the 1- and 5-year cumulative incidence of AP in patients with HNSCC was $15.8 \%$ and $23.8 \%$, respectively. In non-HNSCC patients, these values were $3.6 \%$ and $8.7 \%$, respectively. A multivariate analysis identified several independent risk factors for AP, including hypopharyngeal and nasopharyngeal tumors, male sex, advanced age, increased Charlson Comorbidity Index, no prior surgery to radiation, 
and care received at a teaching hospital. ${ }^{87}$ Several mechanisms involved in the development of AP in patients with HNSCC receiving CCRT have been explored to explain the relationship of this illness with oral radiation. After radiation, AP may develop as the consequence of a series of events induced by acute and chronic mucosal changes, muscle fibrosis, dysphagia, and xerostomia. In turn, these factors lead to swallowing dysfunction, which further increases the risk of both aspiration and aspiration pneumonia. Biological mechanisms involving altered oral conditions and respiratory inflammation have been identified due to oral pathogens directly aspirated into the lungs. ${ }^{88}$ Among them, Pseudomonas aeruginosa has been identified as the most important agent, showing antibiotic resistance and an increased capacity of colonization in patients with NGTs. ${ }^{89}$ Lung injury may be a consequence of the direct destructive effects of these microorganisms on the lung parenchyma, inducing apoptosis of bronchial epithelial cells and an exuberant host immune response. A second mechanism may be represented by the role of salivary enzymes associated with periodontal disease, which modify the mucosal surfaces of the respiratory tract. In such cases, it has been hypothesized that respiratory pathogens promote adhesion and colonization through modification of the mucosal epithelium, loss or removal of surface fibronectin by hydrolytic enzymes, and release of cytokines. ${ }^{90}$ Thus, oral hygiene and radiation-induced dysphagia play a key role in the origin of AP. Poor oral hygiene leads to higher enzymatic activity from periodontopathic bacteria. This higher enzymatic activity destroys the salivary film that protects against pathogenic bacteria through a reduction of the ability of mucins to adhere to pathogens. Consequently, pathogens are able to freely adhere to mucosal receptors in the respiratory tract. ${ }^{91}$ Eventually, the continuous release of cytokines from periodontal tissues and impairment of the immune system of the host amplify the damage resulting in a wide pulmonary infection. Adequate oral care is required to minimize the risk of AP. Regarding dysphagia, assessment of its severity through swallowing tests may be useful to promptly determine the need for the placement of a tube feeding device. ${ }^{92}$

\section{Psychological Distress In Patients With HNC}

Patients with HNSCC are at a high risk of developing emotional problems during all stages of their disease. Therefore, they require a support network that closely follows them during all phases of the disease (ie, from diagnosis to follow-up). Two surveys have been conducted in Western European countries by the European Head and Neck Society. These efforts resulted in the development of a program termed the Make Sense Campaign aiming to raise awareness of the disease and improve outcomes in patients with HNC. ${ }^{93}$ In summary, this program advocates that emotional support should be provided by one designated healthcare professional who monitors the patients during the entire therapeutic journey or in the context of an MDT management approach. Psychological interventions are applied to improve psychological outcomes, increase adherence to treatment protocols, enhance compliance to the treatment, and encourage compliance to lifestyle modifications (eg, consumption of alcohol, use of tobacco, or sexual activities). Moreover, this activity may be useful in recognizing situations requiring pharmacological or psychotherapeutic interventions. In particular, appropriate attention should be paid in the treatment of patients with human papillomavirus-related HNSCC, owing to high risk of psychosocial distress associated with this condition. ${ }^{94}$

\section{Conclusion}

From an oncological and medical point of view, HNSCC is a valid field for the application of a MDT approach to guarantee the best oncological outcome and prevent or adequately treat any adverse effects. Given the complexities of the management of patients with HNSCC, selection of patient and knowledge of competing morbidities is the milestone to advocate and stress the role of the MDT approach. Nutritional counseling, evaluation of swallowing, oral care, and supportive and psychological care management are mandatory prior to, during, and after concomitant chemoradiation therapy. As shown by this review, and consistent with the study conducted by Lo Nigro et al, ${ }^{95}$ the MDT approach should include a radiologist, surgeon, radiation oncologist, medical oncologist, and other professionals involved in supportive care (ie, dietician, dentist, pain and swallowing physician, pneumologist, and psychologist) throughout the entire therapeutic journey to provide the best medical healthcare. Data suggest that the MDT-based approach is beneficial for patients with $\mathrm{HNC}$ and leads to improved survival rates through an improved diagnostic and staging accuracy, a more efficacious therapeutic approach, and enhanced communication across disciplines. 


\section{Disclosure}

The authors report no conflicts of interest in this work.

\section{References}

1. Pignon JP, le Maïtre A, Maillard E, Bourthis J; MACH-NC Collaborative Group. Metanalysis of chemotherapy in Head and neck cancer (MACH-NC): an update on 93randomised trials and 17,346 patients. Radiother Oncol. 2009;92:4-14. doi:10.1016/j. radonc.2009.04.014

2. Forastiere AA, Goepfert H, Maor M, et al. Concurrent chemotherapy and radiotherapy for organ preservation in advanced laryngeal cancer. N Engl J Med. 2003;349:2091-2098. doi:10.1056/NEJMoa031317

3. Ang KK, Chen A, Curran WJ Jr, et al. Head and neck carcinoma in the United States: first comprehensive report of the Longitudinal Oncology Registry of Head and Neck Carcinoma (LORHAN). Cancer. 2012;118:5783-5792. doi:10.1002/cncr.v118.23

4. Bonner JA, Harari PM, Giralt J, et al. Radiochemotherapy plus cetuximab for locoregionally advanced head and neck cancer: 5year survival data from a phase 3 randomised trial, and relation between cetuximab-induced rash and survival. Lancet Oncol. 2010;11(1):21-28. doi:10.1016/S1470-2045(09)70311-0

5. Mell KL, Dignam JJ, Salama JK, et al. Predictors of competing mortality in advanced head and neck cancer. J Clin Oncol. 2010;28 (1):15-20. doi:10.1200/JCO.2008.20.9288

6. Forastiere AA, Zhang Q, Weber RS, et al. Long-term results of RTOG 91-11: a comparison of three nonsurgical treatment strategies to preserve the larynx in patients with locally advanced larynx cancer. J Clin Oncol. 2013;31(7):845-852. doi:10.1200/JCO.2012.43.6097

7. Kwong DL, Sham JS, Chua DT, Choy DT, Au GK, Wu PM. The effect of interruptions and prolonged treatment time in radiotherapy for nasopharyngeal carcinoma. Int J Radiat Oncol Biol Phys. 1997;39:703-710. doi:10.1016/S0360-3016(97)00339-8

8. Slevin NJ, Hendry JH, Roberts SA, Agren-Cronqvist A. The effect of increasing the treatment time beyond three weeks on the control of T2 and T2 laryngeal cancer using radiotherapy. Radiother Oncol. 1992;24:215-220. doi:10.1016/0167-8140(92)90226-K

9. Russi EG, Raber-Durlacher JE, Sonis ST. Local and systemic pathogenesis and consequences of regimen-induced inflammatory responses in patients with head and neck cancer receiving chemoradiation. Mediators Inflamm. 2014;2014:518261. doi:10.1155/2014/ 518261

10. Kwon M, Roh JL, Song J, et al. Non cancer health events as a leading cause of competing mortality in advanced head and neck cancer. Ann Oncol. 2014;25:1208-1214. doi:10.1093/annonc/mdu128

11. Strom TJ, Naghavi AO, Trotti AM, et al. Increased acute mortality with chemoradiotherapy for locally advanced head and neck cancer in patients $\geq 70$ yrs. J Geriatr Oncol. 2017;8(1):50-55. doi:10.1016/j. jgo.2016.09.003

12. Datema FR, Ferrier MB, van der Schroeff MP, Beatenburg de Jong J. Impact of comorbidity on short-term mortality and overall survival of head and neck cancer patients. Head Neck. 2010;32:728-736. doi:10.1002/hed.21245

13. Adelstein D, Gillison ML, Pfister DG, et al. NCCN guidelines insights: head and neck cancer, version 2. J Natl Compr Canc Netw. 2017;15(6):761-770. doi:10.6004/jncen.2017.0101

14. Piccirillo JF, Vlahiotis A. Comorbidity in patients with cancer of the head and neck: prevalence and impact on treatment and prognosis. Curr Oncol Rep. 2006;8(2):123-129. doi:10.1007/s11912-006-0047-z

15. Charlson M, Szatrowski TP, Peterson J, Gold J. Validation of a combined comorbidity index. J Clin Epidemiol. 1994;47:12451251. doi:10.1016/0895-4356(94)90129-5

16. Vitzthum LK, Feng CH, Noticewala S, et al. Comparison of comorbidity and frailty indices in patients with head and neck cancer using an online tool. J Clinical Oncol Clin Cancer Inform. 2018;2:1-9.
17. MacDonald N. Terminology in cancer cachexia: importance and status. Curr Opin Clin Nutr Metab Care. 2012;15:220-225. doi:10.1097/MCO.0b013e328352a 895

18. Roxburgh CS, McMillan DC. Role of systemic inflammatory response in predicting survival in patients with primary operable cancer. Future Oncol. 2010;1:149-163. doi:10.2217/fon.09.136

19. McMillan DC. Systemic inflammation, nutritional status and survival in patients with cancer. Curr Opin Clin Nutr Metab Care. 2009;3:223-226. doi:10.1097/MCO.0b013e32832a7902

20. Proctor MJ, Talwar D, Balmer SM, et al. The relationship between the presence and site of cancer, an inflammation-based prognostic score and biochemical parameters. Initial results of the Glasgow Inflammation Outcome Study. Br J Cancer. 2010;103:870-876. doi:10.1038/sj.bjc. 6605855

21. Chang PH, Wang $\mathrm{CH}$, Chen EYC, et al. Glasgow prognostic score after concurrent chemoradiotherapy is a prognostic factor in advanced head and neck cancer. Chin J Cancer Res. 2017;29 (3):172-178. doi:10.21147/j.issn.1000-9604.2017.03.02

22. Silver HJ, Dietrich M, Murphy BA. Changes in body mass, energy balance, physical function, and inflammatory state in patients with locally advanced head and neck cancer treated with concurrent chemoradiation after low-dose induction chemotherapy. Head Neck. 2007;29:893-900. doi:10.1002/(ISSN)1097-0347

23. Chasen MR, Bhargava R. A descriptive review of the factors contributing to nutritional compromise in patients with head and neck cancer. Support Care Cancer. 2009;17:1345-1351. doi:10.1007/s00 520-009-0684-5

24. Pauloski BR, Rademaker AW, Logemann JA, Discekici-Harris M, Mittal BB. Comparison of swallowing function after intensity-modulated radiation therapy and conventional radiotherapy for head and neck cancer. Head Neck. 2015;37:1575-1582. doi:10.1002/hed.v37.11

25. Ottosson S, Zackrisson B, Kjellén E, Nillson P, Laurell J. Weight loss in patients with head and neck cancer during and after conventional and accelerated radiotherapy. Acta Oncol. 2013;52:711-718. doi:10.3109/0284186X.2012.731524

26. Capuano G, Grosso A, Gentile PC, et al. Influence of weight loss on outcomes in patients with head and neck cancer undergoing concurrent chemoradiotherapy. Head Neck. 2008;30:5503-5508. doi:10.10 02/hed.20737

27. Bole'o-Tome C, Monteiro-Grillo I, Camilo M, Ravasco P. Validation of the Malnutrition Universal Screening Tool (MUST) in cancer. British J Nutr. 2012;108:343-348. doi:10.1017/S000711451100571X

28. Datema FR, Ferrier MB, Baatenburg de Jong RJ. Impact of severe malnutrition on short-term mortality and overall survival in head and neck cancer. Oral Oncol. 2011;47(9):910-914. doi:10.1016/j. oraloncology.2011.06.510

29. Arends J, Bodoky G, Bozzetti F, et al. ESPEN Guidelines on Enteral Nutrition, Non-surgical oncology. Clin Nutr. 2006;25:245-259. doi:10.1016/j.clnu.2006.01.020

30. Bauer DJ, Capra S. Nutrition support using the American Dietetic Association medical nutrition therapy protocol for radiation oncology patients improves dietary intake compared with standard practice. $J$ Am Diet Assoc. 2007;107:404-412. doi:10.1016/j.jada.2006.12.007

31. Ash S, Davidson LW, Hill MJ, et al. Evidence based practice guidelines for the nutritional management of patients receiving radiation therapy of the Dietitians Association of Australia. Nutr Diet. 2008;65:1-20.

32. Paccagnella A, Morello M, Da Mosto MC, et al. Early nutritional intervention improves treatment tolerance and outcome in head and neck cancer patients undergoing concurrent chemoradiotherapy. Support Care Cancer. 2010;18:837-845. doi:10.1007/s00520-009-0717-0

33. Hughes BGM, Jain VK, Brown T, et al. Decreased hospital stay and significant cost savings after routine use of prophylactic gastrostomy for high-risk patients with head and neck cancer receiving chemoradiotherapy at a tertiary cancer institution. Head Neck. 2013;35 (3):436-442. doi:10.1002/hed.22992 
34. Bradley PT, Brown T, Paleri V. Gastrostomy in head and neck cancer: current literature, controversies and research. Curr Opin Otolaryngol Head Neck Surg. 2015;23:162-170. doi:10.1097/MOO.000000000 0000135

35. Talwar B, Donnelly R, Skelly R, Donaldson M. Nutritional management in head and neck cancer: United Kingdom National Multidisciplinary guidelines. J Laryngol Otol. 2016;130(Supp12):3240. doi:10.1017/S0022215116000402

36. Brawn T, Ross L, Jones L, Hughes B, Banks M. Nutrition outcomes following implementation of validated swallowing and nutrition guidelines for patients with head and neck cancer. Support Care Cancer. 2014;22:2381-2391. doi:10.1007/s00520014-2180-9

37. Chao KS, Deasy JO, Markman J, et al. A prospective study of salivary function sparing in patients with head -and -neck cancers receiving intensity-modulated or three dimensional radiotherapy: initial results. Int J Radiat Oncol Biol Phys. 2001;49:907-916. doi:10.1016/S0360-3016(00)01441-3

38. Kondrup J, Rasmussen HH, Hamberg O, Stanga Z; Ad Hoc, ESPEN Working Group. Nutritional risk screening (NRS 2002): a new method based on an analysis of controlled clinical trials. Clin Nutr. 2003;22:321-336. doi:10.1016/S0261-5614(02)00214-5

39. Corry J, Poon W, McPhee N, et al. Prospective study of percutaneous endoscopic gastrostomy tubes for enteral feeding in patients with head and neck cancer undergoing (chemo)radiation. Head Neck. 2009;31:867-876. doi:10.1002/hed.21044

40. Nugent B, Lewis S, O'Sullivan JM. Enteral feeding methods for nutritional management in patients with head and neck cancers being treated with radiotherapy and/or chemotherapy. Cochrane Database Syst Rev. 2013;31(1):CD007904.

41. Wang J, Liu M, Liu C, Ye Y, Huang G. Percutaneous endoscopic gastrostomy versus nasogastric tube feeding for patients with head and neck cancer: a systematic review. J Radiat Res. 2014;55(3):559_ 567. doi:10.1093/jrr/rrt144

42. Bossola M. Nutritional interventions in head and neck cancer patients undergoing chemoradiotherapy: a narrative review. Nutrients. 2015;7:265-276. doi:10.3390/nu7010265

43. Lewis SL, Brody R, Touger-Decker R, Parrott JS, Epstein J. Feeding tube use in head and neck cancer patients. Head Neck. 2014;36 (12):1789-1795. doi:10.1002/hed.23538

44. Baschnagel AM, Yadav S, Marina O, et al. Toxicities and costs of placing prophylactic and reactive percutaneous gastrostomy tubes in patients with locally advanced head and neck cancers treated with chemoradiotherapy. Head Neck. 2014;36:1155-1161. doi:10.1002/ pro.v36.8

45. Strom TJ, Trotti AM, Kish J, et al. Risk factors for percutaneous endoscopic gastrostomy tube placement during chemoradiotherapy for oropharyngeal cancer. JAMA Otolaryngol Head Neck Surg. 2013;139(11):1242-1246. doi:10.1001/jamaoto.2013. 5193

46. Irvine D, Vincent L, Graydon JE, Bubela N, Thompson L. The prevalence and correlates of fatigue in patients receiving treatment with chemotherapy and radiotherapy. A comparison with the fatigue experienced by healthy individuals. Cancer Nurs. 1994;17:367-378. doi:10.1097/00002820-199410000-00001

47. Hickok JT, Roscoe JA, Morrow GR, Mustian K, Okunieff P, Bole CW. Frequency, severity, clinical course, and correlates of fatigue in 327 patients during 5 weeks of radiotherapy for cancer. Cancer. 2005;104(8):1772-1778. doi:10.1002/cncr.21364

48. Jereczek-Fossa BA, Santoro L, Alterio D, et al. Fatigue during headand-neck radiotherapy: prospective study on 117 consecutive patients. Int $J$ Radiat Oncol Biol Phys. 2007;68(2):403-415. doi:10.1016/j.ijrobp.2007.01.024

49. Biswal BM, Kuaraswamy N, Mukhtar F. Prevalence of fatigue among cancer patients undergoing external radiotherapy. Southeast Asian J Trop Med Public Health. 2004;35:463-467.
50. Brown DJ, McMillan DC, Milroy R. The correlation between fatigue, physical function the systemic inflammatory response, and psychological distress in patients with advanced lung cancer. Cancer. 2005;103:377-382. doi:10.1002/cncr.20777

51. Dicato M, Duhem C, Berchem G, Ries F. Clinical benefit from erythropoietin. Curr Opin Oncol. 2000;12:297-302. doi:10.1097/ 00001622-200007000-00004

52. Hoskin PJ, Robinson M, Slevin N, Morgan D, Harrington K, Gaffney C. Effect of epoetin alfa on survival and cancer treatment-related anemia and fatigue in patients receiving radical radiotherapy with curative intent for head and neck cancer. J Clin Oncol. 2009;27 (34):5751-5756. doi:10.1200/JCO.2009.22.3693

53. Bunn HF. Anemia. In: Harrison's Principles of Internal Medicine. 10th ed. McGrow-Hill; 1983:54.

54. Bohlius J, Weingart O, Trelle S, Engert A. Cancer-related anemia and recombinant human erythropoietin-an updated overview. Nat Clin Pract Oncol. 2006;3(3):152-164. doi:10.1038/ncponc0451

55. Spivak JL. The anaemia of cancer: death by a thousand cuts. Nat Rev Cancer. 2005;5:543-555.

56. $\mathrm{Hu} \mathrm{K}$, Harrison LB. Impact of anemia in patients with head and neck cancer treated with radiation therapy. Curr Treat Options Oncol. 2005;6(1):31-35. doi:10.1007/s11864-005-0011-4

57. Ghadjar P, Pöttgen C, Joos D, et al. Haemoglobin and creatinine values as prognostic factors for outcome of concurrent radiochemotherapy in locally advanced head and neck cancers: secondary results of two European randomized phase III trials (ARO 95-06, SAKK 10/94). Strahelenthe rOnkol. 2016;192(8):552-560. doi:10.1007/s00066-016-0999-3

58. Henke M, Laszig R, Rübe C, et al. Erythropoietin to treat head and neck cancer patients with anaemia undergoing radiotherapy: randomized, double-blind, placebo-controlled trial. Lancet. 2003;362: 1255-1260. doi:10.1016/S0140-6736(03)14567-9

59. Overgaard J, Hoff CM, Hansen HS, et al. DAHANCA 10 - Effect of darbepoetin alfa and radiotherapy in the treatment of squamous cell carcinoma of the head and neck. A multicenter, open-label, randomized, phase 3 trial by the Danish head and neck cancer group. Radiother Oncol. 2018;127(1):12-19. doi:10.1016/j.radonc.2018.02. 018

60. Shenouda G, Zhang Q, Ang KK, et al. Long-term results of radiation therapy oncology group 9903: a randomized phase 3 trial to assess the effect of erythropoietin on local-regional control in anemic patients treated with radiation therapy for squamous cell carcinoma of the head and neck. Int J Radiat Oncol Biol Phys. 2015;91(5):907915. doi:10.1016/j.ijrobp.2014.12.018

61. Aapro MS, Link H. September 2007 Update on EORTC Guidelines and Anemia Management with Erythropoiesis-Stimulating Agents. Oncologist. 2008;13(3):33-36. doi:10.1634/theoncologist.13-S3-33

62. Bohlius J, Bohlke K, Castelli R, et al. Management of cancer-associated anemia with erythropoiesis-stimulating agents: ASCO/ASH clinical practice guideline update. Blood Adv. 2019;3(8):1197-1210. doi:10.1182/bloodadvances.2018030387

63. Quon H, Leong T, Haselow R, Leipzig B, Cooper J, Forastiere A. Phase III study of radiation therapy with or without cisplatinum in patients with unresectable squamous or undifferentiated carcinoma oh the head and neck: an intergroup trial of the Eastern Cooperative Oncology Group. Int J Radiat Oncol Biol Phys. 2011;81:719-725. doi:10.1016/j.ijrobp.2010.06.038

64. Espeli V, Zucca E, Ghielmini M, Giannini O, Salatino A, Richetti A. Weekly and 3-weekly cisplatin concurrent with intensity-modulated radiotherapy in locally advanced head and neck squamous cell cancer. Oral Oncol. 2012;48:266-271. doi:10.1016/j.oraloncology.2011.10.005

65. Rades D, Seidi D, Janssen S, et al. Chemoradiation of locally advanced squamous cell carcinoma of the head and neck (LASCCHN): is $20 \mathrm{mg} / \mathrm{m}$ (2) cisplatin on five days every four weeks an alte rnative to $100 \mathrm{mg} / \mathrm{m} \mathrm{(2)} \mathrm{cisplatin} \mathrm{every} \mathrm{three} \mathrm{weeks?}$ Oral Oncol. 2016;59:67-72. doi:10.1016/j.oraloncology.2016.06.004 
66. Strojan P, Vermorken JB, Beitler JJ, et al. Cumulative cisplatin dose in concurrent chemoradiotherapy for head and neck cancer: a systematic review. Head Neck. 2016;38(1):2151-2158. doi:10.1002/ hed. 24026

67. Bernier J, Domenge C, Ozsahin M, et al. Postoperative irradiation with or without concomitant chemotherapy for locally advanced head and neck cancer. N Engl J Med. 2004;350(19):1945-1952. doi:10. 1056/NEJMoa032641

68. Cooper JS, Pajak TF, Forastiere AA, et al. Postoperative concurrent radiotherapy and chemotherapy for high-risk squamous-cell carcinoma of the head and neck. N Engl J Med. 2004;350(19):19371944. doi:10.1056/NEJMoa032646

69. Grunberg SM, Warr D, Gralla RJ, et al. Evaluation of new antiemetic agents and definition of antineoplastic agent emetogenicity: stare of the art. Support Care Cancer. 2011;19(suppl1):43-47.

70. National Comprehensive Guidelines Network. NCCN clinical practice guidelines in oncology: antiemesis-v2. 2018. Available from: https//www.nccn.org/professionals/physician_gls/default.aspx. Accessed October 30, 2019.

71. Herrstedt J, Roila F, Warr D, et al. Updated MASCC/ESMO consensus recommendations: prevention of nausea and vomiting following high emetic risk chemotherapy. Support Care Cancer. 2017;251:277-288. doi:10.1007/s00520-016-3313-0 2016.

72. Hesketh PJ, Kris MG, Basch E, et al. Antiemetics: American society of clinical oncology clinical practice guideline update. J Clin Oncol. 2017;35(28):3240-3261. doi:10.1200/JCO.2017.74.4789

73. Pabla N, Dong Z. Cisplatin nephrotoxicity: mechanisms and renoprotective strategies. Kidney Int. 2008;73:994-1007. doi:10.1038/sj. ki.5002786

74. Hoek J, Blomendal KM, van der Velden LAA, et al. Nephrotoxicity as a dose-limiting factor in a high-dose cisplatin -based chemoradiotherapy regimen for head and neck carcinomas. Cancers. 2016;8 (21):1-9. doi:10.3390/cancers8020021

75. Ludwig H, Van Belle S, Barrett-Lee P, et al. The European Cancer Anaemia Survey (ECAS): a large multinational, prospective survey defining the prevalence, incidence, and treatment of anaemia in cancer patients. Eur J Cancer. 2004;40(15):2293-2306. doi:10.10 16/j.ejca.2004.06.019

76. Caro JJ, Salas M, Ward A, Goss G. Anemia as an independent prognostic factor for survival in patients with cancer: a systemic, quantitative review. Cancer. 2001;91:2214-2221. doi:10.1002/(ISSN) 1097-0142

77. Wood PA, Hrushesky JM. Cisplatin -associated anemia: an Erythropoietin Deficiency Syndrome. J Clin Invest. 1995;95:16501659. doi:10.1172/JCI117840

78. Stroop WG, Schaefer DC. Production of encephalitis restricted to the temporal lobes by experimental reactivation of herpes simplex virus. J Infect Dis. 1986;153(4):72. doi:10.1093/infdis/153.4.721

79. Zhang MY, Zhu GQ, Shi KQ, et al. Systematic review with network meta-analysis: comparative efficacy of oral nucleos(t)ide analogues for the prevention of chemotherapy-induced hepatitis B virus reactivation. Oncotarget. 2016;21(7):30642-30658.

80. Klastersky J, Aoun M. Opportunistic infections in patients with cancer. Ann Oncol. 2004;15(4):329-335.
81. Singh GK, Capoor MR, Nair D, Bhowmik KT. Spectrum of fungal infection in head and neck cancer patients on chemoradiotherapy. $J$ Egypt Natl Canc Inst. 2017;29:33-37. doi:10.1016/j.jnci.2017.01.006

82. Wingard JR, Merz WG, Rinaldi MG, Johnson TR, Karp JE, Saral R. Increase in Candida Krusei infection among patients with bone marrow transplantation and neutropenia treated prophylactically with fluconazole. N Engl J Med. 1991;325(18):1274-1277. doi:10.1056/ NEJM199110313251803

83. Piper JP, Rinaldi MG, Winn ME. Candida Parapsilosis: an emerging problem. Inf Dis Newsl. 1988;7:49-56.

84. Redding SW, Dahiya MC, Kirkpatrick WR, et al. Candida glabrata is an emerging cause of oropharyngeal candidiasis in patients receiving radiation for head and neck cancer. Oral Surg Oral Med Oral Pathol Oral Radiol Endo. 2004;2097(1):47-52. doi:10.1016/j.tripleo.2003. 09.008

85. Marik PE. Aspiration Pneumonitis and aspiration pneumonia. $N$ Engl J Med. 2001;344:665-671. doi:10.1056/NEJM200103013440908

86. Mortensen HR, Jensen K, Grau C. Aspiration pneumonia in patients treated with radiotherapy for head and neck cancer. Acta Oncol. 2013;52:270-276. doi:10.3109/0284186X.2012.742205

87. Beibei X, Boero IJ, Hwang L, et al. Aspiration pneumonia after concurrent chemoradiotherapy for head-and-neck cancer. Cancer. 2015;121(8):1303-1311.

88. Gomes-Filho I, Passos J, Seixas da Cruz S. Respiratory disease and the role of oral bacteria. J Oral Microbiol. 2010;21:2.

89. Leibovitz A, Dan M, Zinger J, Carmeli Y, Habot B, Segal R. Pseudomonas aeruginosa and the oropharyngeal ecosystem of tubefed patients. Emerg Infect Dis. 2003;9:956-959. doi:10.3201/eid0 908.030054

90. Grassme H, Kirschnek S, Riethmueller J, et al. CD95/CD95 ligand interactions on epithelial cells in host defense to Pseudomonas aeruginosa. Science. 2000;290:527-530. doi:10.1126/science.290.5491. 527

91. Langmore SE, Terpenning MS, Schork A, et al. Predictors of aspiration pneumonia: how important is dysphagia? Dysphagia. 1998;13 (2):69-81. doi:10.1007/PL00009559

92. Lazzari G, De Cillis MA, Terlizzi A, Silvano G. Swallowing tests and aspiration pneumonia in advanced head and neck cancer: preliminary data. Poster presented at 7 th International Conference of Head and Neck ICHNO; March 14-16; 2019; Barcelona (Spain). doi:10.1016/ S0167-8140(19)30308-1

93. Reich M, Leemans CR, Vermorken JB, et al. Best practices in the management of the psycho-oncologic aspects of the head and neck cancer patients: recommendations from the European Head and Neck Cancer Society Make Sense Campaign. Ann Oncol. 2014;25:21152124. doi:10.1093/annonc/mdu105

94. Baxi SS, Shuman AG, Corner GW, et al. Sharing a diagnosis of HPVrelated head and neck cancer: the emotions, the confusion, and what patients want to know. Head Neck. 2013;35:1534-1541. doi:10.1002/ hed.v35.11

95. Lo Nigro C, Denaro N, Merlotti A, Merlano M. Head and neck cancer: improving outcomes with a multidisciplinary approach. Cancer Manag Res. 2017;9:363-371. doi:10.2147/CMAR

\section{Publish your work in this journal}

Cancer Management and Research is an international, peer-reviewed open access journal focusing on cancer research and the optimal use of preventative and integrated treatment interventions to achieve improved outcomes, enhanced survival and quality of life for the cancer patient.
The manuscript management system is completely online and includes a very quick and fair peer-review system, which is all easy to use. Visit http://www.dovepress.com/testimonials.php to read real quotes from published authors. 